\title{
Processo de Trabalho na Estratégia de Saúde da Família: Perspectiva dos Cirurgiões-Dentistas do Distrito Federal ${ }^{1}$
}

\author{
Work Process in the Family Health Strategy: Perspective of Dental \\ Surgeons of the Federal District
}

Procedimiento de Trabajo en la Estrategia de Salud de la Familia:

Perspectiva de los Cirujanos Dentistas del Distrito Federal

Regina Cardoso de Moura², Tiago Araújo Coelho de Souza ${ }^{3}$

\section{Resumo}

O objetivo deste estudo transversal, descritivo e inferencial foi analisar o processo de trabalho quanto à integralidade em saúde e integração em equipe na perspectiva dos cirurgiões-dentistas da Estratégia de Saúde da Família (ESF). Foi aplicado um questionário eletrônico, com escala para as perguntas variando entre 0 e 10 pontos. Foi utilizada estatística descritiva para apresentação das características da amostra; teste de Pearson para análises associativas; teste $\mathrm{t}$ de student para amostras independentes e ANOVA oneway para comparações entre grupos; nível de significância de $5 \%(\mathrm{p}<0,05)$. No processo de trabalho relacionado à integralidade, o atendimento às urgências odontológicas e o acolhimento à demanda espontânea tiveram as melhores avaliações e a visita domiciliar a menor. No processo de trabalho relacionado à integração com a equipe, o compartilhamento da agenda foi o mais bem avaliado. Correlação positiva fraca entre educação permanente e ações regulares de prevenção $(\mathrm{r}=0,489$; $\mathrm{p}<0,001)$, ações regulares de promoção $(\mathrm{r}=0,468 ; \mathrm{p}<0,001)$ e atenção integral $(\mathrm{r}=0,344 ; \mathrm{p}=0,001)$ foram observadas. Os profissionais que não possuem vínculo à uma equipe da ESF ou que cumprem 20 horas apresentaram avaliações mais baixas em ações de integralidade e integração, impactando negativamente na organização e gestão dos processos de trabalho da saúde bucal na ESF no Distrito Federal.

\footnotetext{
1 Trabalho de Conclusão de Curso no Programa de Pós-graduação lato sensu - Residência Multiprofissional em Saúde Coletiva. Título original: "INTEGRALIDADE E INTEGRAÇÃO DA SAÚDE BUCAL NA ESTRATÉGIA DE SAÚDE DA FAMÍLIA: PERCEPÇÃO DOS CIRURGIÕES-DENTISTAS DA SECRETARIA DE ESTADO DE SAÚDE DO DISTRITO FEDERAL". Escola Superior de Ciências da Saúde, Brasília-DF, 2018.

${ }^{2}$ Cirurgiã-Dentista, Especialista em Saúde Coletiva pela Residência Multiprofissional em Saúde Coletiva (Escola Superior em Ciências da Saúde ESCS), Especialista em Odontopediatria (ABO Seção Goiás), graduada pela Universidade de Brasília (UnB). Gerente na Gerência de Acesso e Qualidade da Atenção Primária, Diretoria Regional de Atenção Primária da Região de Saúde Norte, Secretaria de Estado de Saúde do Distrito Federal.

${ }^{3}$ Cirurgião-Dentista; Doutor em Saúde Pública pela University of Kentucky, Mestre em Odontologia pela Universidade Federal de Minas Gerais, graduado pela Universidade Federal do Pará. Professor do Departamento de Odontologia da Faculdade de Ciências da Saúde da Universidade de Brasília. E-mail: tiagosouza@unb.br
} 
Revista Eletrônica Gestão \& Saúde ISSN: 1982-4785 Moura RC, Souza TAC

Descritores: Saúde Bucal; Prática Profissional; Estratégia Saúde da Família.

\section{Abstract}

The purpose of this cross-sectional, descriptive and inferential study was to analyze the work process regarding integrality in health and team integration from the perspective of dental surgeons of the Family Health Strategy (FHS). An electronic questionnaire was applied, with a scale ranging from 0 to 10 points. Descriptive statistics to present the characteristics of the sample, Pearson test for associative analysis, Student's $\mathrm{t}$ test for independent samples and one-way ANOVA for comparisons between groups were used; level of significance of $5 \%$ $(\mathrm{p}<0.05)$. In the work process related to integrality, attendance to dental emergencies and response to spontaneous demand had the best evaluations; home visits showed the worse evaluation. In the work process related to integration with the team, the shared agenda was the best evaluated. Positive weak correlation between continuing education and regular oral health prevention actions $(\mathrm{r}=0,489 ; \mathrm{p}<0,001)$, regular promotion actions $(\mathrm{r}=0,468 ; \mathrm{p}<0,001)$, and comprehensive oral health care were observed $(r=0,344 ; p=0,001)$. Dentists who do not have a bond with an FHS team or who work only 20 hours a week presented lower evaluations on actions related to integrality and integration, therefore causing Rev. Gestão \& Saúde (Brasília) Vol. 10, № 2. Maio, 2019.
Processo de Trabalho na Estratégia de Saúde...

a negative impact on the organization and management of oral health work processes in the FHS in the Federal District.

Key Words: Oral Health; Professional Practice; Family Health Strategy

\section{Resumen}

El objetivo de este estudio transversal, descriptivo e inferencial fue analizar el proceso de trabajo en cuanto a la integralidad en salud e integración en equipo en la perspectiva de los cirujanos-dentistas de la Estrategia de Salud de la Familia (ESF). Se aplicó un cuestionario electrónico, con escala para las preguntas variando entre 0 y 10 puntos. Se utilizó estadística descriptiva para la presentación de las características de la muestra; prueba de Pearson para análisis asociativos; prueba $\mathrm{t}$ de student para muestras independientes y ANOVA una forma para comparaciones entre los grupos; nivel de significancia de 5\% (p $<0,05)$. En el proceso de trabajo relacionado a la integralidad, la atención a las urgencias odontológicas y la acogida a la demanda espontánea tuvieron las mejores evaluaciones y la visita domiciliar, la menor. En el proceso de trabajo relacionado a la integración en equipo, el compartir de la agenda fue el mejor evaluado. Se observó correlación positiva débil entre educación permanente y acciones regulares de prevención $(r=0,489 ; \mathrm{p}<0,001)$, promoción $(\mathrm{r}=0,468 ; \mathrm{p}<0,001)$ y atención integral $(r=0,344 ; p=0,001)$. Los profesionales 
Revista Eletrônica Gestão \& Saúde ISSN: 1982-4785 Moura RC, Souza TAC

que no tienen vínculo a un equipo o que trabajan 20 horas presentaron peores evaluaciones en las acciones, impactando negativamente en la organización y la gestión de los procesos de trabajo.

Descriptores: Salud Bucal; Práctica Profesional; Estrategia de Salud de la Familia

\section{Introdução}

Entre os atributos essenciais da Atenção Primária à Saúde (APS) estão o processo de trabalho, o acesso, a longitudinalidade, a integralidade e a coordenação do cuidado. Entende-se que, a partir desses atributos prioritários, um serviço de atenção primária de qualidade precisará ter a sua 'porta de entrada' facilitada ao usuário e um fluxo de atenção à saúde organizado ${ }^{(1)}$.

A criação da Estratégia Saúde da Família, em 1994, trouxe a reorganização da lógica assistencial, com a focalização na família como unidade de ação programática de saúde, e não mais a centralização no indivíduo ou na doença ${ }^{(2)}$. Apesar do Programa de Saúde da Família (PSF) ter sido implantado em 1994, a incorporação da saúde bucal deu-se apenas em 2000, com a Portaria Ministerial $n^{\circ}$ $1.444^{(3)}$.

Com a inclusão da equipe de Saúde Bucal (eSB) na equipe de Saúde da Família (eSF), a odontologia se viu imersa em um processo de mudança de paradigma, em uma nova ótica de atenção à saúde, a que busca Rev. Gestão \& Saúde (Brasília) Vol. 10, № 2. Maio, 2019.
Processo de Trabalho na Estratégia de Saúde...

visualizar o paciente como um todo e não apenas os seus problemas bucais ${ }^{(4)}$. No entanto, tendo em vista o histórico diverso de atuação dos cirurgiões-dentistas das eSB inseridos nas eSF no Brasil, bem como a carência de estudos avaliativos sobre a inserção desses profissionais no novo contexto de atuação, se faz imprescindível analisar 'quais' mudanças e 'como' realmente estão ocorrendo nas práticas em nível local.

Nesse contexto, este estudo teve como objetivo analisar o processo de trabalho quanto à integralidade em saúde e integração em equipe na perspectiva dos cirurgiões-dentistas da Estratégia de Saúde da Família no Distrito Federal.

\section{Referencial Teórico}

De acordo com a atual Política Nacional de Atenção Básica ${ }^{(5)}$, a Saúde da Família é a estratégia de atenção à saúde prioritária para expansão e reorganização da Atenção Básica. Favorece a reorganização do processo de trabalho para ampliação da resolutividade, de forma a impactar na situação de saúde de pessoas e coletividades. Nesse contexto, busca-se a produção de uma atenção integral com a promoção e prevenção em saúde, diagnóstico, tratamento, reabilitação e redução de danos e sofrimentos.

A Equipe de Saúde da Família é composta por, no mínimo, médico, enfermeiro, auxiliar e/ou técnico de enfermagem e agente 
comunitário de saúde. Podem fazer parte da equipe o agente de combate às endemias e os profissionais de saúde bucal, o cirurgiãodentista e o auxiliar ou técnico em saúde bucal $^{(5)}$.

Dentro do processo de trabalho das equipes da Atenção Básica, devem estar presentes as seguintes características: definição do território e territorialização; responsabilização sanitária; adscrição de usuários e vinculação; acesso; acolhimento; trabalho em equipe multiprofissional; resolutividade; promoção de atenção integral; ações de atenção domiciliar; programação e atenção à saúde; promoção da saúde; ações de prevenção de doenças e agravos; ações educativas; ações intersetoriais; planejamento local de saúde; formação e educação permanente em saúde ${ }^{(5)}$.

No que concerne ao trabalho em equipe multiprofissional, o alcance do atendimento integral se dá a partir da integração das diversas áreas técnicas profissionais, ao se desenvolverem ações compartilhadas e interdisciplinares de saúde centradas no usuário, somando-se vigilância, promoção e assistência. No processo de trabalho interdisciplinar, devem ser incorporadas também a clínica ampliada e o matriciamento, podendo-se utilizar, entre outras estratégias, a construção de Projeto Terapêutico Singular e trabalho com grupos, de acordo com as necessidades da população ${ }^{(5)}$.
A participação em reuniões de equipe é uma importante atribuição comum a todos os profissionais da Atenção Básica e objetiva a readequação constante do processo de trabalho, ao proporcionar o planejamento e a avaliação sistemática das ações da equipe com a utilização dos dados disponíveis ${ }^{(5)}$.

Nesse contexto, a saúde bucal é vinculada à uma equipe de Saúde da Família e deve compartilhar com esta a gestão e o processo de trabalho, tendo a mesma população, território adstrito e responsabilidade sanitária ${ }^{(5)}$.

A formação e Educação Permanente em Saúde, também prevista no processo de trabalho das equipes, é entendida como a aprendizagem desenvolvida no trabalho, capaz de transformar as práticas dos trabalhadores de saúde. Devem fazer parte do cotidiano das equipes, ter espaço garantido na carga horária de trabalho e abarcar todas os profissionais ${ }^{(5)}$.

Conforme ALMEIDA FILHO (2013, p. 1681)* discorre em um artigo de comentário introdutório, o modelo hegemônico de formação superior em saúde no Brasil ainda é privatizante, de prática especialista e centrada no hospital. Esse modelo não atende às necessidades de saúde da população, tampouco às necessidades do SUS. Ressalta-se o desafio da formação de trabalhadores que atuam na saúde brasileira engajados com o trabalho em equipe, na promoção da saúde, na participação social e na humanização da atenção à saúde. 
Revista Eletrônica Gestão \& Saúde ISSN: 1982-4785 Moura RC, Souza TAC

\section{Metodologia}

A presente pesquisa, de desenho descritivo e inferencial transversal, teve autorização e suporte da Gerência de Odontologia da Secretaria de Estado de Saúde do Distrito Federal (SES-DF) para estabelecimento de contato, por meio eletrônico, com os cirurgiões-dentistas da ESF. Para tal, foi desenvolvido um questionário autoavaliativo com base em dois instrumentos do Programa de Melhoria do Acesso e Qualidade da Atenção Básica (PMAQ-AB) do Ministério da Saúde, a saber: a) "Ficha de informações para o profissional de nível superior" constante no Instrumento de Avaliação Externa para as Equipes de Atenção Básica e Saúde Bucal $^{(6)}$ do PMAQ; e b) “Autoavaliação para a Melhoria do Acesso e da Qualidade da Atenção Básica” (AMAQ) $)^{(7)}$.

A escolha do AMAQ como um dos instrumentos-referência se deu pelo fato deste ser norteado pelos princípios e diretrizes da Atenção Básica, partindo do pressuposto de que todos os gestores municipais e equipes de Atenção Básica podem e devem utilizá-lo ${ }^{(7)}$. As dimensões de interesse do AMAQ visando à construção do instrumento foram: JEducação permanente e qualificação das Equipes de Saúde Bucal; K- Organização do processo de trabalho; L- Atenção Integral à Saúde Bucal. Assim como no AMAQ, foi utilizada uma escala de pontuação para as perguntas, variando entre 0 e 10 pontos.
Processo de Trabalho na Estratégia de Saúde...

Ao utilizar o instrumento, o respondente teve que considerar o ponto 0 (zero) como indicando 'não cumprimento ao padrão' e o ponto 10 como de 'total adequação ao padrão', sendo os intervalos entendidos como 'graus de conformidade/atendimento da

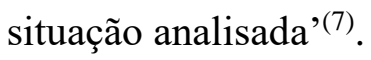

As perguntas constantes do questionário sofreram adaptações para adequálas à aplicação eletrônica, assim como algumas perguntas foram divididas para avaliar informações separadamente. Foram também inseridas perguntas acerca da 'Organização do processo trabalho', não contempladas no instrumento original.

Foi realizado um pré-teste com cinco cirurgiões-dentistas da Gerência de Odontologia da SES, com experiência em Atenção Primária, com o intuito de avaliar o entendimento, o roteiro elaborado e a adequação das perguntas antes de sua aplicação final. Após as correções e adaptações necessárias, iniciou-se a coleta de dados.

Os profissionais receberam por e-mail um convite com informações acerca da pesquisa e, caso aceitassem, poderiam acessar link específico para responder ao questionário. $\mathrm{O}$ método descrito foi escolhido devido à possibilidade de alcance de grande número de profissionais e, ao mesmo tempo, pela facilidade de acesso ao questionário no tempo 
Revista Eletrônica Gestão \& Saúde ISSN: 1982-4785 Moura RC, Souza TAC

do respondente, pela velocidade em compilar os dados e pela redução de custos.

No momento do estudo, 277 cirurgiões-dentistas faziam parte do quadro da Atenção Primária à Saúde da Secretaria de Estado de Saúde do Distrito Federal. Os critérios de inclusão utilizados foram: a) ser cirurgião-dentista; b) atuar em Equipe de Saúde Bucal da Estratégia de Saúde da Família no Distrito Federal; c) ter aceitado e respondido a todo o questionário de auto avaliação aplicado eletronicamente. Foram excluídos da análise de dados os cirurgiõesdentistas que relataram atuar em período integral ou parcial em cargo de gestão na SESDF, bem como os questionários de pré-teste.

Para permitir melhor descrição dos resultados, as médias das notas obtidas na auto avaliação foram categorizadas, de acordo com as escalas do $\mathrm{AMAQ}^{(6)}$, em: a) 0 a 2 pontos 'muito insatisfatório'; b) 3 a 4 pontos 'insatisfatório'; c) 5 a 6 pontos 'regular'; d) 7 a 8 pontos 'satisfatório'; e) 9 a 10 pontos 'muito satisfatório'.

Nas variáveis analisadas, são variáveis dependentes as notas autoavaliativas dos profissionais em cada dimensão analisada (educação permanente, processo de trabalho e atenção integral à saúde bucal). São variáveis independentes no presente estudo os dados sociodemográficos dos profissionais (sexo, idade, tempo de conclusão da graduação, presença e tipo de pós-graduação realizada), os

\section{Processo de Trabalho na Estratégia de Saúde...}

dados estruturais dos serviços e de organização da atenção primária (região de saúde onde atua, carga horária de trabalho, tempo de atuação na eSB, vínculo a uma eSF e tempo de vinculação à eSF).

Os dados com todas as respostas dos profissionais foram consolidados em planilha Excel® e foi utilizada estatística descritiva e inferencial para apresentação do perfil e formação dos cirurgiões-dentistas, os quais foram expressos em média e desvio-padrão ou em frequência absoluta e relativa. $\mathrm{O}$ pressuposto de normalidade foi testado por meio do teste Shapiro-Wilk. Para verificar se havia correlação das notas autoavaliativas de 'Educação Permanente' com as notas autoavaliativas de 'Processo de Trabalho' e 'Integralidade em Saúde Bucal' de cada participante adotou-se o teste de correlação de Pearson. Para as comparações das notas autoavaliativas dos profissionais agrupados de acordo com tipo de perfil e formação, foram utilizados os testes $\mathrm{t}$ de student para amostras independentes e ANOVA one-way. O nível de significância adotado foi de $5 \%(\mathrm{p}<0,05)$ e todas as análises foram conduzidas no software Statistical Package for Social Sciences (SPSS) versão 22.0.

Este estudo seguiu as normas da Resolução n 196/96 do Conselho Nacional de Saúde e recebeu parecer favorável (CAAE: 81655417.6.0000.5553) do Comitê de Ética e 
Revista Eletrônica Gestão \& Saúde ISSN: 1982-4785 Moura RC, Souza TAC

Pesquisa da Fundação de Ensino e Pesquisa em Ciências da Saúde/ FEPECS/SES/ DF.

\section{Resultados}

Após os critérios de exclusão, 89 profissionais permaneceram na amostra, o que representa $32,1 \%$ do total de cirurgiõesdentistas (CDs) da Atenção Básica da SES-DF. A média de idade dos CDs respondentes foi de $41,6 \operatorname{anos}(\mathrm{DP}=5,7)$.

Como pode ser observado na tabela 1 , a maioria dos CDs pesquisados exercem 40 horas, têm entre 5 a 10 anos de tempo de graduação e têm alguma pós-graduação. Dentre esses profissionais que têm pósgraduação, apenas 31,5\% (n=28) concluíram pós-graduação lato sensu em Saúde da Família, Saúde Pública ou Saúde Coletiva, área de formação com aplicação direta ao cargo desses profissionais. No que concerne à vinculação da equipe de saúde bucal a uma equipe de Saúde da Família, 85,4\% (n=76) dos CDs atuam em uma equipe vinculada a uma equipe de Saúde da Família.

Nas perguntas de autoavaliação, a primeira etapa era relativa à 'Educação Permanente'. A tabela 2 descreve as médias e desvios-padrão das respostas dos participantes.

Nas respostas categorizadas para a dimensão apresentada, as perguntas 2.1, 2.3,
Processo de Trabalho na Estratégia de Saúde...

2.4 e 2.5 apresentaram maior frequência de respostas classificadas em 'satisfatório' e/ou 'muito satisfatório'. Já a pergunta 2.6 teve maior frequência de respostas $(56,2 \%)$ classificadas em 'muito insatisfatório' e também apresentou a menor média geral, 2,73. Em seguida, a pergunta 2.2 apresentou maior frequência de respostas classificadas em 'muito insatisfatório', ‘insatisfatório' e 'regular' e média menor que 5,0.

A segunda etapa de perguntas autoavaliativas foi acerca do 'Processo de Trabalho', subdividido, neste estudo, em 'Processo de trabalho relacionado à Integralidade' e 'Processo de Trabalho para Integração à eSB'.

A tabela 3 descreve as médias e desvios-padrão das respostas dos participantes. Nas respostas categorizadas, as questões 3.1, 3.4, 3.6, 3.10, 3.11, 3.12 apresentaram maior frequência de respostas classificadas em 'satisfatório' ou 'muito satisfatório' e as maiores médias dentre as perguntas dessa dimensão. As perguntas 3.7 e 3.9 apresentaram maior frequência de respostas classificadas em 'muito insatisfatório' ou 'insatisfatório' dentre todas acerca de 'Processo de trabalho'. Essas perguntas também apresentaram as menores médias, conforme pode ser visto na tabela 2 . 
Tabela 1 - Características da amostra de profissionais pesquisada

\begin{tabular}{|c|c|c|c|}
\hline Variável & Percentual (\%) & Variável & Percentual (\%) \\
\hline \multicolumn{2}{|l|}{ Sexo } & \multicolumn{2}{|c|}{ Pós-graduação na área de atuação } \\
\hline Feminino & 74,2 & Lato Sensu & 35,0 \\
\hline \multirow[t]{2}{*}{ Masculino } & 25,8 & Stricto Sensu & 6,3 \\
\hline & & Outra área & 58,8 \\
\hline \multicolumn{4}{|l|}{ Região de Saúde } \\
\hline Centro-Norte & 3,4 & Tempo de atuação & \\
\hline Norte & 19,1 & Até 2 anos & 7,9 \\
\hline Centro-Sul & 10,1 & 2 a 5 anos & 10,1 \\
\hline \multirow[t]{2}{*}{ Sul } & 13,5 & 5 a 10 anos & 51,7 \\
\hline & & Mais de 10 anos & 30,3 \\
\hline \multicolumn{4}{|l|}{ Carga horária } \\
\hline 20 horas & 18,0 & Vinculação à eSF & \\
\hline \multirow[t]{2}{*}{40 horas } & 82,0 & Sim & 85,4 \\
\hline & & Não & 14,6 \\
\hline \multicolumn{4}{|c|}{ Tempo de graduação } \\
\hline 1 a 5 anos & 6,7 & Tempo de atuação na ESF & \\
\hline 5 a 10 anos & 93,3 & Até 2 anos & 49,4 \\
\hline & & 2 a 5 anos & 17,2 \\
\hline Pós-graduação & & 5 a 10 anos & 31,0 \\
\hline Sim & 91,0 & Mais de 10 anos & 2,3 \\
\hline Não & 9,0 & & \\
\hline
\end{tabular}

Fonte: Os autores, 2018 


\section{Tabela 2 - Autoavaliação da Educação Permanente que qualifica para a Atenção Básica}

\begin{tabular}{|c|c|c|}
\hline Perguntas & Média & $\mathrm{DP} *$ \\
\hline 2.1 - Formação complementar que qualifica para a Atenção Básica (AB) & 6,72 & 2,58 \\
\hline 2.2 - Oferta, pela Secretaria de Estado de Saúde (SES), de cursos de Atualização, & 4,61 & 2,63 \\
\hline \multicolumn{3}{|l|}{ Aperfeiçoamento e Qualificação (AAQ) para a AB } \\
\hline 2.3 - Participação em cursos de AAQ oferecidos pela SES & 6,21 & 3,10 \\
\hline 2.4 - Participação em cursos de AAQ com recursos próprios & 6,38 & 2,89 \\
\hline 2.5 - Participação em cursos à distância para qualificação do cuidado & 5,71 & 3,53 \\
\hline 2.6 - Utilização de dispositivos de Apoio Matricial à distância para qualificação do cuidado: & 2,73 & 2,95 \\
\hline \multicolumn{3}{|l|}{ Teleconsultorias, Segunda Opinião Formativa, Fóruns de Discussão em Comunidade de } \\
\hline Práticas, entre outros. & & \\
\hline
\end{tabular}

* DP = Desvio Padrão; Fonte: Os autores, 2018

A última etapa da autoavaliação, 'Atenção Integral à Saúde Bucal', é descrita na tabela 4, com as médias e desvios-padrão das respostas dos participantes. Nas respostas categorizadas, algumas questões apresentaram maior frequência de respostas classificadas em 'satisfatório' ou 'muito satisfatório', tais como as perguntas 4.1, 4.2 e 4.6. Essas perguntas também apresentaram as maiores médias dentre as perguntas dessa dimensão. A única questão com maior frequência de respostas classificadas como 'muito insatisfatório' foi a pergunta 4.5 , que também teve a menor das médias dessa dimensão, 4,94 $(\mathrm{DP}=3,07)$.

$\mathrm{Na}$ análise de correlação das notas autoavaliativas dos participantes quanto às dimensões 'Educação Permanente' e ‘Atenção
Integral à Saúde Bucal', observou-se correlação positiva fraca entre as duas dimensões $\quad(r=0,344 ; \quad p=0,001)$. Essa correlação positiva fraca permeou todas as análises entre as perguntas realizadas, a saber: a) oferta de cursos de atualização, aperfeiçoamento e qualificação, e realização de ações regulares de prevenção em saúde bucal $(r=0,383 ; p<0,001)$; b) participação em cursos de atualização, aperfeiçoamento e qualificação, e realização de ações regulares de prevenção em saúde bucal ( $\mathrm{r}=0,347 ; \mathrm{p}=0,001)$; c) participação em cursos à distância para qualificação do cuidado e realização de ações regulares de prevenção em saúde bucal $(\mathrm{r}=0,322 ; \mathrm{p}=0,002) ; \mathrm{d})$ utilização de dispositivos de apoio matricial para qualificação do cuidado e realização de ações regulares de prevenção em saúde bucal 
Revista Eletrônica Gestão \& Saúde ISSN: 1982-4785 Moura RC, Souza TAC

$(\mathrm{r}=0,320 ; \mathrm{p}=0,002) ; \mathrm{e})$ ações regulares de promoção de saúde e oferta de cursos de atualização, aperfeiçoamento e qualificação $(\mathrm{r}=0,324 ; \mathrm{p}=0,002) ; \mathrm{f})$ ações regulares de promoção de saúde e participação em cursos de atualização, aperfeiçoamento e qualificação $(\mathrm{r}=0,338 ; \mathrm{p}=0,001) ; \mathrm{g})$ ações regulares de promoção de saúde e participação em cursos à distância para qualificação do cuidado $(\mathrm{r}=0,320 ; \mathrm{p}=0,002)$.

$\mathrm{Na}$ análise de correlação das notas autoavaliativas quanto às dimensões 'Educação Permanente' e 'Processo de trabalho', observou-se correlação positiva moderada entre as duas dimensões $(r=0,431$; $\mathrm{p}=0,000)$. Nas análises entre as perguntas realizadas observou-se correlação positiva fraca, a saber: a) Formação complementar que qualifica para a Atenção Básica e monitoramento e análise de indicadores de saúde bucal da população do território ( $r=0,324 ; p=0,002)$; b) Participação em cursos de Atualização, Aperfeiçoamento e Qualificação oferecidos pela SES e monitoramento e análise de ações e resultados ( $r=0,336 ; p=0,001)$; ) Participação em cursos à distância para qualificação do cuidado e atuação para integração dos demais profissionais da eSF em discussão de casos e projetos terapêuticos $(\mathrm{r}=0,362 ; \mathrm{p}=0,000) ; \mathrm{d})$ formação complementar que qualifica para a
Processo de Trabalho na Estratégia de Saúde...

Atenção Básica e organização da carga horária semanal para atenção clínica individual, visita domiciliar, educação em saúde, acolhimento, ações de vigilância, ações coletivas, reuniões de planejamento e avaliação ( $r=0,325$; $\mathrm{p}=0,002)$; e) formação complementar que qualifica para a Atenção Básica e implementação de estratégias para correção rumos a partir dos resultados alcançados $(\mathrm{r}=0,321 ; \mathrm{p}=0,002)$.

A comparação das notas autoavaliativas se deu com relação ao perfil dos profissionais agrupados por Regiões de Saúde, carga horária desenvolvida na Atenção Básica, tempo de conclusão da graduação, pósgraduação concluída, tipo de pós-graduação realizada, tempo de atuação na $\mathrm{AB}$, vínculo com a eSF e tempo na eSF atual. A seguir serão descritas as comparações que apresentaram diferenças significativas entre os grupos.

Nas notas dos profissionais agrupados pelas Regiões de Saúde do DF, houve diferença significativa $(p=0,03)$ na pergunta 'Participação em reuniões periódicas com a equipe de Saúde da Família' da Região Sul $(4,25 ; \mathrm{DP}=3,75)$ com a Região Leste $(8,67$; $\mathrm{DP}=0,86$ ). Houve também diferença significativa $(\mathrm{p}=0,03)$ nas notas da pergunta 'Realização de ações regulares de promoção de saúde bucal na rede pública de ensino' da Região Oeste $(7,58 ; \mathrm{DP}=2,50)$ com a Região 
Revista Eletrônica Gestão \& Saúde ISSN: 1982-4785 Moura RC, Souza TAC

$\operatorname{Sul}(4,33 ; \mathrm{DP}=2,60)$. Também houve diferença significativa $(\mathrm{p}=0,03)$, na mesma pergunta, da Região Oeste $(7,58 ; \mathrm{DP}=2,50)$ com a Região Leste $(4,00 ; \mathrm{DP}=2,78)$.

A comparação das notas autoavaliativas entre grupos de carga horária realizadas pelos cirurgiões-dentistas da APS mostrou diferença significativa em questões
Processo de Trabalho na Estratégia de Saúde...

relativas a 'Processo de trabalho', tanto no que se refere à integralidade quanto integração à eSF (tabela 4). Também houve diferença estatisticamente significativa $(p=0,04)$ para a soma total das médias das respostas de 'Processo de Trabalho'.

Tabela 3 - Autoavaliação do Processo de Trabalho e Integralidade

\begin{tabular}{|c|c|c|}
\hline Perguntas & Média & DP* \\
\hline \multicolumn{3}{|l|}{ Dimensão: Processo de trabalho relacionado à Integralidade } \\
\hline 3.1 - Trabalho com Territorialização, Vinculação e Responsabilização. & 7,37 & 2,68 \\
\hline 3.2 - Planejamento de ações pelo perfil de risco do território e envolvimento da comunidade. & 5,61 & 2,59 \\
\hline 3.3 - Monitoramento e análise de indicadores de saúde bucal da população do território. & 4,48 & 2,40 \\
\hline 3.5 - Realização da coordenação do cuidado em saúde bucal. & 6,84 & 2,44 \\
\hline 3.6 - Compreensão da abordagem familiar e adoção de uma concepção de família ampliada. & 7,88 & 2,07 \\
\hline $\begin{array}{l}3.7 \text { - Utilização de estratégias/ferramentas de abordagem familiar e de grupos sociais } \\
\text { específicos. }\end{array}$ & 3,51 & 2,45 \\
\hline $\begin{array}{l}3.8 \text { - Organização da carga horária semanal para: atenção clínica individual, visitas } \\
\text { domiciliares, grupos de educação em saúde, acolhimento, ações de vigilância, ações coletivas, } \\
\text { reuniões de planejamento/avaliação. }\end{array}$ & 5,65 & 2,59 \\
\hline 3.9 - Realização de visita domiciliar sistemática, programada e oportuna. & 3,62 & 2,50 \\
\hline 3.10 - Realização de acolhimento à demanda espontânea em tempo integral. & 8,28 & 2,11 \\
\hline 3.11 - Garantia de atendimento a todas as urgências odontológicas. & 8,97 & 1,17 \\
\hline 3.12 - Referência de urgências de casos que não consegue atender. & 7,83 & 2,70 \\
\hline 3.15 - Utilização de informações do sistema de informação para qualificar a atenção. & 4,73 & 2,95 \\
\hline 3.16 - Monitoramento e análise de ações e resultados. & 4,18 & 2,55 \\
\hline 3.17 - Implementação de estratégias para correção de rumos a partir dos resultados alcançados. & 4,47 & 2,69 \\
\hline 3.18 - Registro e monitoramento de encaminhamento e retorno de especialidades & 6,37 & 2,83 \\
\hline \multicolumn{3}{|l|}{ Dimensão: Processo de trabalho para integração à eSF } \\
\hline 3.4 - Compartilhamento de agenda de atendimento individual com os outros profissionais. & 7,01 & 2,58 \\
\hline 3.13 - Participação em reuniões periódicas com a equipe de Saúde da Família. & 6,74 & 3,24 \\
\hline
\end{tabular}


3.14 Temas de discussão na reunião de equipe: organização do processo de trabalho, planejamento, educação permanente, troca de experiências, discussão de casos, projeto $6,22 \quad 3,18$ terapêutico singular ou planejamento de consultas compartilhadas.

3.19 Atuação para integração dos demais profissionais da eSF em discussão de casos e projetos terapêuticos.

Dimensão: Atenção Integral à Saúde Bucal

4.1- Realização de ações regulares de prevenção em saúde bucal na população adscrita.

$6,79 \quad 2,52$

4.2 - Realização de ações regulares de promoção em saúde bucal na população adscrita.

4.3 - Realização de ações regulares de prevenção em saúde bucal na rede pública de ensino.

4.4 - Realização de ações regulares de promoção em saúde bucal na rede pública de ensino.

\section{Tabela 4 - Análise bivariada entre a carga-horária de trabalho e variáveis do processo de trabalho relacionado à integralidade}

\begin{tabular}{|c|c|c|c|}
\hline \multicolumn{3}{|c|}{ Parte I: Processo de trabalho relacionado à Integralidade } & p-valor \\
\hline \multicolumn{4}{|c|}{ 3.1 Trabalho com: Territorialização, Vinculação e Responsabilização } \\
\hline & Média & $\mathrm{DP}^{*}$ & \\
\hline CD 20h & 5,56 & 3,70 & $\mathrm{p}=0,04$ \\
\hline $\mathrm{CD} 40 \mathrm{~h}$ & 7,77 & 2,24 & \\
\hline
\end{tabular}

3.2 Planejamento de ações de acordo com perfil de risco do território e envolvimento da comunidade.

Média DP

$\begin{array}{lrrr}\text { CD 20h } & 4,25 & 2,97 & \mathrm{p}=0,02 \\ \mathrm{CD} 40 \mathrm{~h} & 5,90 & 2,41 & \end{array}$

3.3 Monitoramento e análise de indicadores de saúde bucal da população do território. Média DP

$\begin{array}{llll}\text { CD 20h } & 3,31 & 2,70 & \mathrm{p}=0,03 \\ \mathrm{CD} 40 \mathrm{~h} & 4,74 & 2,27 & \end{array}$

3.7 Utilização de estratégias/ferramentas de abordagem familiar e de grupos sociais específicos. $\quad \mathrm{p}=0,01$ 
Revista Eletrônica Gestão \& Saúde ISSN: 1982-4785

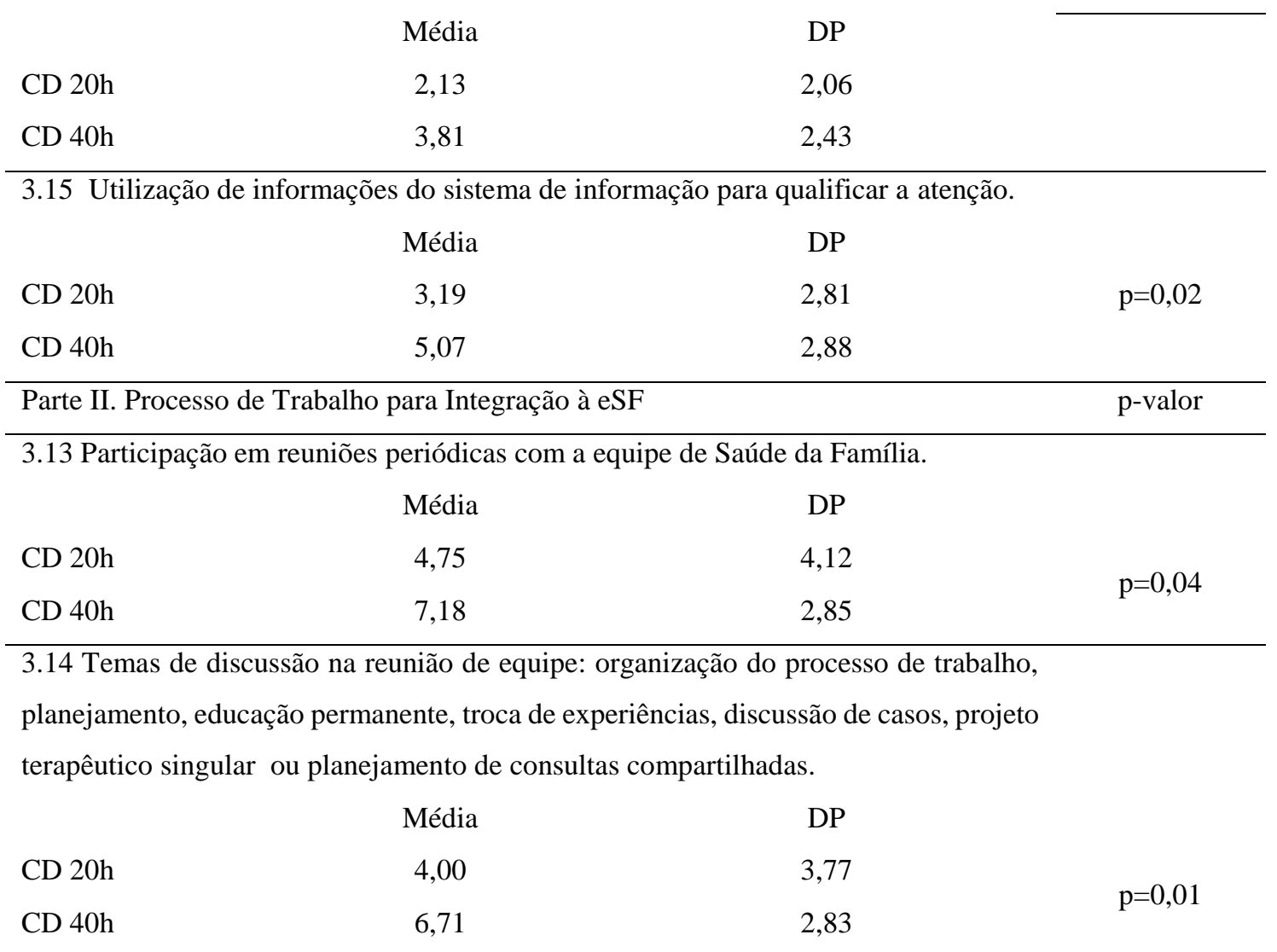

\begin{tabular}{lccc}
\hline 3.19 Atuação para integração dos demais profissionais da eSF em discussão de casos & \\
e projetos terapêuticos. & Média & DP & \\
& 4,69 & 3,68 & $\mathrm{p}=0,04$ \\
CD 20h & 6,78 & 2,55 & \\
CD 40h & Média & DP & \\
\hline Soma total - Processo de trabalho & 40,83 & $\mathrm{p}=0,04$ \\
& 97,63 & 27,83 & \\
CD 20h & 120,23 & 23 & \\
CD 40h &
\end{tabular}

*DP= Desvio Padrão; Fonte: Os autores, 2018.

Na comparação das médias das notas autoavaliativas entre os dois grupos relativos a tempo de graduação dos CDs, houve diferença significativa $(\mathrm{p}=0,04)$ na questão de 'Processo de Trabalho' de 'Compartilhamento de agenda de atendimento individual com os outros profissionais', em que o grupo de 1-5 anos apresentou média de 5,00 (DP=2,60) e o grupo de 5-10 anos, média de 7,16 (DP=2,53). Nas notas referentes à 'Atenção Integral em Saúde 
Revista Eletrônica Gestão \& Saúde ISSN: 1982-4785 Moura RC, Souza TAC

Bucal', houve diferença significativa $(\mathrm{p}=0,04)$ para a soma total das médias das respostas dos CDs que possuem 1-5 anos e 5-10 anos de graduação.

A comparação das médias das notas autoavaliativas de quem possui ou não pósgraduação mostrou diferença significativa $(\mathrm{p}=0,04)$ apenas para uma pergunta de 'Processo de Trabalho' relacionado à Integralidade: 'Utilização de informações do sistema de informação para qualificar a atenção'. Entre os profissionais que possuem alguma pós-graduação a média foi de 4,93 (DP=2,91) e entre os que não possuem a média foi de 2,75 (DP=2,71).

A comparação das médias das notas autoavaliativas entre o grupo de profissionais que se vinculavam à uma eSF e o dos que não se vinculavam mostrou diferença significativa nas perguntas de
Processo de Trabalho na Estratégia de Saúde...

'Processo de Trabalho' relacionadas à integralidade e integração à eSF, conforme pode ser visto na tabela 5 , e também para a soma total de notas de 'Processo de Trabalho'. Também houve diferença significativa nas notas de duas questões de 'Atenção Integral à Saúde Bucal'. Na questão de 'Ações regulares de promoção em saúde bucal na rede pública de ensino’ houve diferença significativa $(\mathrm{p}=0,04)$ entre quem possui vínculo $(6,21 ; \mathrm{DP}=2,85)$ e quem não possui $(4,46 ; \mathrm{DP}=3,07)$, assim como na questão de 'Realização de ações de avaliação em saúde bucal na rede pública de ensino', $(p=0,04)$, entre quem possui vínculo $(5,21 ; \mathrm{DP}=3,05)$ e quem não possui $(3,38 ; \mathrm{DP}=2,72)$.

Tabela 5 - Análise bivariada entre a carga-horária de trabalho e variáveis do processo de trabalho relacionado à integralidade.

\begin{tabular}{|c|c|c|c|}
\hline \multicolumn{3}{|c|}{ Parte I: Processo de trabalho relacionado à Integralidade } & \multirow[t]{2}{*}{ p-valor } \\
\hline 3.1 Trabc & ação, Vi & zação & \\
\hline & Média & $\mathrm{DP}^{*}$ & \\
\hline CD 20h & 5,56 & 3,70 & \\
\hline $\mathrm{CD} 40 \mathrm{~h}$ & 7,77 & 2,24 & $\mathrm{p}=0,04$ \\
\hline
\end{tabular}

3.2 Planejamento de ações de acordo com perfil de risco do território e envolvimento da comunidade.

Média DP

$\begin{array}{lll}\text { CD 20h } & 4,25 & 2,97\end{array}$

$2,97 \quad \mathrm{p}=0,02$


Revista Eletrônica Gestão \& Saúde ISSN: 1982-4785

CD 40h

5,90

2,41

3.3 Monitoramento e análise de indicadores de saúde bucal da população do território.

$\begin{array}{cccc} & \text { Média } & \text { DP } & \\ \text { CD 20h } & 3,31 & 2,70 & \\ \text { CD 40h } & 4,74 & 2,27 & \mathrm{p}=0,03\end{array}$

3.7 Utilização de estratégias/ferramentas de abordagem familiar e de grupos sociais específicos.

Média DP

$\begin{array}{llll}\text { CD 20h } & 2,13 & 2,06 & \mathrm{p}=0,01 \\ \mathrm{CD} 40 \mathrm{~h} & 3,81 & 2,43 & \end{array}$

3.15 Utilização de informações do sistema de informação para qualificar a atenção.

Média DP

$\begin{array}{llll}\text { CD 20h } & 3,19 & 2,81 & \mathrm{p}=0,02 \\ \text { CD 40h } & 5,07 & 2,88 & \end{array}$

Parte II. Processo de Trabalho para Integração à eSF p-valor

3.13 Participação em reuniões periódicas com a equipe de Saúde da Família.

Média DP

$\begin{array}{llll}\text { CD 20h } & 4,75 & 4,12 & \mathrm{p}=0,04 \\ \mathrm{CD} 40 \mathrm{~h} & 7,18 & 2,85 & \end{array}$

3.14 Temas de discussão na reunião de equipe: organização do processo de trabalho, planejamento, educação permanente, troca de experiências, discussão de casos, projeto terapêutico singular ou planejamento de consultas compartilhadas.

\begin{tabular}{lccc} 
& Média & DP & \\
CD 20h & 4,00 & 3,77 & $\mathrm{p}=0,01$ \\
CD 40h & 6,71 & 2,83 & \\
\hline
\end{tabular}

3.19 Atuação para integração dos demais profissionais da eSF em discussão de casos e projetos terapêuticos.

$\begin{array}{cccc} & \text { Média } & \text { DP } & \\ \text { CD 20h } & 4,69 & 3,68 & \mathrm{p}=0,04 \\ \text { CD 40h } & 6,78 & 2,55 & \end{array}$

\begin{tabular}{lccc}
\hline \multicolumn{2}{l}{ Soma total - Processo de trabalho } & & \\
& Média & DP & \\
CD 20h & 97,63 & 40,83 & $\mathrm{p}=0,04$ \\
CD 40h & 120,23 & 27,83 &
\end{tabular}

* $\overline{\mathrm{DP}}=$ Desvio Padrão; Fonte: Os autores, 2018. 
Revista Eletrônica Gestão \& Saúde ISSN: 1982-4785 Moura RC, Souza TAC

\section{Discussão}

Os resultados encontrados neste estudo mostram que os cirurgiões-dentistas avaliados apresentam conhecimento satisfatório dentro de vários atributos essenciais para atuação na atenção primária, porém ainda existem fragilidades a serem superadas. Nesse sentido, $\operatorname{Reis}^{(8)}$ discute a necessidade do redirecionamento do processo de trabalho devido às novas competências exigidas do trabalhador de saúde, trazendo à tona a necessidade de formação do trabalhador do SUS, em especial para a saúde da família. Entendendo que este estudo apresentou limitações por ser transversal com ênfase quantitativa e ter resultados inferenciais, recomenda-se que investigações qualitativas e longitudinais sejam realizadas para melhor compreender as fraquezas, a fim de nortear a gestão em suas ações e tomada de decisão com os profissionais estudados.

O percentual de respostas ao questionário autoavaliativo obtido neste estudo representa uma taxa de resposta dentro do esperado para pesquisas realizadas por meio eletrônico ${ }^{(9)}$.

A maioria dos profissionais relatou possuir alguma pós-graduação em odontologia, porém a maioria dos profissionais possui esse nível de formação em áreas da odontologia distintas da Saúde Pública, Saúde
Processo de Trabalho na Estratégia de Saúde...

Coletiva ou Saúde da Família. Ao discutir acerca do perfil formativo do CD brasileiro, Narvai ${ }^{(10)}$ indica a inadequação do preparo dos profissionais, uma vez que são formados de forma desvinculada das reais necessidades do país e são direcionados de forma precoce para especialidades, além da inadequação para os serviços onde deverão atuar. Apesar dessas informações, não houve diferença nas notas autoavaliativas ao se comparar o grupo com pós-graduação direcionada para atuação na atenção primária com o grupo com pósgraduação em outras áreas.

A maioria dos profissionais relatou possuir entre 5 e 10 anos de graduação e uma pequena parcela menos de 5 anos. A comparação das médias das notas entre os dois grupos de CDs mostrou diferença significativa em questões acerca da 'Atenção Integral à Saúde Bucal', com maiores médias para os profissionais com menos de 5 anos de formados. Em um estudo em que os CDs apresentavam no mínimo cinco anos de formados, foi apontada a diferente realidade do ensino da época como fator determinante para as dificuldades enfrentadas atualmente no trabalho na $\operatorname{ESF}^{(11)}$. Essa pode ser uma das explicações para a diferença das notas no presente estudo.

Com relação à carga horária de trabalho, a maioria dos CDs que responderam 
Revista Eletrônica Gestão \& Saúde ISSN: 1982-4785 Moura RC, Souza TAC

à pesquisa realizam 40 horas semanais, porém ainda está presente uma parcela de profissionais que realizam 20 horas. Além disso, na comparação entre grupos de CDs que cumprem 20 horas e 40 horas na APS, houve diferença significativa para um número elevado de questões relativas a 'Processo de trabalho' relacionado a integralidade e a integração à eSF, demonstrando que a carga horária que o profissional realiza também impacta em suas ações com a população e com a equipe na qual trabalha. A singularidade de existirem cargos de 20 horas para a APS, presente no Distrito Federal, contrasta com o que é definido pelo Ministério da Saúde, ao prever a carga horária de 40 horas para os profissionais das equipes de saúde ${ }^{(5)}$.

A Educação Permanente que qualifica para a Atenção Básica mostrou resultados favoráveis para as questões que se referem à formação complementar que o profissional considera possuir e/ou participar. Em um estudo $^{(3)}$ que avaliou os CDs da APS, mais de 90\% afirmaram ter participado de cursos de capacitação. No entanto, a utilização de dispositivos de apoio matricial à distância, tais como teleconsultorias, segunda opinião formativa e fóruns de discussão em comunidade de práticas, mostrou o pior desempenho na 'Educação Permanente' e a menor média geral desse estudo. Esse

\section{Processo de Trabalho na Estratégia de Saúde...}

resultado poderia ser esperado, tendo em vista que o núcleo da Rede Telessaúde Brasil ainda está em fase de implantação no Distrito Federal, sendo necessária maior divulgação no contexto local.

Quando consideradas as variáveis 'Educação Permanente' e 'Processo de Trabalho', foi observada correlação positiva fraca entre perguntas relativas à integralidade e uma pergunta relativa a integração à eSF. Também houve correlação positiva fraca das notas de 'Educação Permanente' com as notas de 'Atenção Integral em Saúde Bucal' dos profissionais, referentes à promoção e prevenção de saúde. Esses resultados demostram necessidade acerca do aumento na qualificação profissional no que tange ao conhecimento do seu processo de trabalho na ESF. Destaca-se, assim, a necessidade de maior oferta de cursos destinados a esses profissionais, levando ainda em consideração que a oferta pela SES-DF de cursos de Atualização, Aperfeiçoamento e Qualificação para a $\mathrm{AB}$ não foi considerada satisfatória pela maioria dos CDs avaliados.

Na dimensão 'Processo de Trabalho' relacionado a Integralidade, muitas questões apresentaram boas notas, referidas pelos profissionais. $\mathrm{O}$ acolhimento às demandas espontâneas em tempo integral, atendimento a todas as urgências odontológicas, referência de 
Revista Eletrônica Gestão \& Saúde ISSN: 1982-4785 Moura RC, Souza TAC

urgências que não consegue atender e coordenação do cuidado foram alguns dos temas das questões de destaque. O Caderno de Atenção Básica $n^{\circ} 17$ destaca a importância do acolhimento da demanda espontânea ao afirmar que todos os serviços de saúde bucal devem ser organizados para acolher essa demanda e os casos de urgência, dando respostas às necessidades da população e sendo um caminho fundamental para a resolutividade da atenção ${ }^{(12)}$.

A territorialização, vinculação e responsabilização, além da abordagem com conceito de família ampliada, também foram temas das questões de destaque. O território e a população, além da dinâmica familiar e social, são parte da rotina de trabalho das Equipes de Saúde da Família e oferecem informações valiosas para o planejamento, acompanhamento e avaliação das ações ${ }^{(5)}$.

No que se refere às notas da dimensão 'Processo de trabalho' relacionado à integralidade na ESF, uma das perguntas com menor desempenho diz respeito à realização de visita domiciliar sistemática, programada e oportuna. Nessa questão, 66,3\% dos profissionais se autoavaliaram entre 'muito insatisfatório' e 'insatisfatório'. Esse resultado se aproxima dos achados de um estudo que analisou os dados da Avaliação Externa do primeiro ciclo do PMAQ, referentes à
Processo de Trabalho na Estratégia de Saúde...

realização de visita domiciliar ${ }^{(13)}$. Nele foi encontrado que $56,1 \%$ dos profissionais da saúde bucal, cirurgiões-dentistas e técnicos de saúde bucal, não realizam cuidado domiciliar, com resultados bem inferiores em relação aos demais profissionais pesquisados.

Outra questão com notas inferiores diz respeito à utilização de estratégias/ferramentas de abordagem familiar e de grupos sociais específicos. Nessa questão, 66,2\% dos profissionais se autoavaliaram entre ‘insatisfatório' e 'muito insatisfatório'. Um estudo que analisou a percepção da equipe de saúde bucal sobre o paciente em seu contexto familiar e comunitário, com a utilização do Genograma e Ciclo de Vida Familiar, constatou que essas ferramentas ainda são pouco utilizadas. Nesse mesmo estudo, apesar de $50 \%$ dos cirurgiões-dentistas pesquisados realizarem atendimento familiar, apenas $20,9 \%$ faziam uso de Genograma ${ }^{(14)}$. Tais ferramentas poderiam ser mais utilizadas na Estratégia Saúde da Família para auxiliar na aproximação dos profissionais com as famílias, aspecto essencial para o êxito da estratégia. Uma possível causa para esse resultado pode ser a falta de conhecimento dos profissionais quanto à existência dessas ferramentas e sua aplicação, o que pode ser confirmado com mais estudos. O Núcleo Ampliado de Saúde da Família (NASF) pode 
Revista Eletrônica Gestão \& Saúde ISSN: 1982-4785 Moura RC, Souza TAC

ter um importante papel na capacitação desses profissionais no uso dessas ferramentas.

Dentro de 'Processo de trabalho' para Integração à eSF, teve destaque positivo a questão relativa ao compartilhamento de agenda com demais profissionais. A atual Política Nacional de Atenção Básica reforça que é fundamental que o processo de trabalho na Atenção Básica inclua uma agenda de trabalho compartilhada de todos os profissionais, de forma a evitar a divisão de agenda e a dificuldade de acesso dos usuários $^{(5)}$.

$\mathrm{Na}$ dimensão 'Atenção Integral à Saúde Bucal', a realização de ações regulares de prevenção e de promoção em saúde bucal na população do território adscrito foi autoavaliada entre 'satisfatório' e 'muito satisfatório'. O resultado encontrado nesse estudo se contrapõe a outros estudos transversais que avaliaram o processo de trabalho dos CDs da Saúde da Família e demonstraram dificuldades por parte dos CDs na realização de ações de prevenção e promoção da saúde ${ }^{(3,15)}$; também apontaram que as atividades da equipe de saúde bucal com a comunidade estão mais voltadas para ações de prevenção e promoção de saúde em creches e escolas ${ }^{(3,16)}$. Porém, no presente estudo, as ações de prevenção e promoção para escolares, na rede pública, demonstraram menor
Processo de Trabalho na Estratégia de Saúde...

desempenho que as ações para a população do território adstrito como um todo, o que gera a necessidade de um olhar mais específico para essa realidade. Esse estudo não abordou os aspectos qualitativos em que a prevenção e promoção em saúde bucal são realizadas, o que pode ser objeto de outras pesquisas.

As questões sobre avaliação em saúde bucal, monitoramento e análise de indicadores de ações e resultados apresentaram algumas das menores médias em suas dimensões, no presente trabalho. Outros estudos indicaram baixa realização de levantamentos pela saúde bucal na atenção primária, a fim de reconhecer as necessidades da população ${ }^{(13,15)}$. Maiores investigações são necessárias para demonstrar quais dificuldades são enfrentadas pelos cirurgiões-dentistas ao realizarem avaliação e monitoramento da situação de saúde bucal de sua população, tendo em vista ser essa uma potente ferramenta para programar as ações da equipe.

A pergunta relativa à 'integralidade da atenção a todas as faixas etárias e gêneros de toda a população do território' apresentou elevada frequência de notas classificadas entre 'satisfatório' e 'muito satisfatório'. Outro estudo, porém, quando investigou a integralidade relacionada ao acesso à atenção em saúde bucal, encontrou disparidades no acesso referente a grupos e segmentos 
Revista Eletrônica Gestão \& Saúde ISSN: 1982-4785 Moura RC, Souza TAC

populacionais com maior vulnerabilidade social, tais como pessoas de baixa renda e idosos $^{(17)}$. Sendo essa realidade passível de ser estendida ao contexto local do DF, o entendimento do conceito de integralidade de atenção e acesso por parte dos profissionais pode ser alvo de maior exploração.

Outro dado importante encontrado nesse estudo refere-se ao fato de que, mesmo que a grande maioria dos CDs da APS estejam em uma equipe de saúde bucal vinculada a uma equipe de Saúde da Família, os CDs relataram trabalhar em equipes não vinculadas. Nesse estudo, a comparação entre grupos de CDs que são vinculados ou não à uma também mostrou diferença significativa nas médias das notas em questões de 'Processo de trabalho' além de questões relativas à 'Atenção Integral à Saúde Bucal'. Isso demonstra que a vinculação da equipe de saúde bucal com uma equipe de Saúde da Família impacta em suas ações integrais com a população e integração com a equipe na qual trabalha. Apesar de no DF a Política de Atenção Primária à Saúde vigente ser fundamentada na Estratégia Saúde da Família, tal política prevê que a equipe de Saúde Bucal será responsável por um território equivalente, no máximo, ao de duas equipes de Saúde da Família $^{(18)}$. Essa previsão se diferencia também da Política Nacional de Atenção Básica em vigor ${ }^{(5)}$. Esses resultados
Processo de Trabalho na Estratégia de Saúde...

reforçam a concepção ideal de uma equipe de Saúde Bucal vinculada a apenas uma equipe de Saúde da Família, de forma a se trabalhar com territorialização e maior conhecimento da comunidade assistida.

As notas dos CDs das Regiões de Saúde mostraram diferença significativa em duas questões. Essas comparações podem não revelar todos os aspectos envolvidos, uma vez que as Regiões de Saúde do Distrito Federal possuem características distintas e precisam ser analisadas de acordo com as mesmas. Recomenda-se que a gestão das respectivas Regiões de Saúde direcione um olhar específico para as fragilidades e potencialidades apontadas nesse estudo.

\section{Conclusão}

Os cirurgiões-dentistas avaliaram como satisfatórias várias questões relacionadas à integralidade e integração na Estratégia de Saúde da Família (ESF). Outro ponto forte apontado diz respeito à Educação Permanente, já que apresentaram boas notas em atualização, aperfeiçoamento e qualificação para a Atenção Básica, o que demonstra o interesse dos cirurgiões-dentistas em se atualizarem. Além disso, o aumento de notas da Educação Permanente repercutiu positivamente nas notas dos profissionais relacionadas à integralidade e integração na 
Revista Eletrônica Gestão \& Saúde ISSN: 1982-4785 Moura RC, Souza TAC

eSF. Destaca-se também que os cirurgiõesdentistas que cumprem 40 horas semanais ou que possuem vínculo com uma equipe de Saúde da Família demonstraram maiores notas paras ações de integralidade com a população e integração com a equipe na qual trabalha.

As fraquezas apontadas pelos profissionais dizem respeito à prevenção e promoção em saúde bucal na rede pública de ensino, ao cuidado domiciliar e à utilização de estratégias e ferramentas de abordagem familiar e grupos sociais específicos. Os cirurgiões-dentistas que não possuem vínculo com uma equipe da ESF ou que cumprem 20 horas apresentaram avaliações mais baixas em ações de integralidade e integração, impactando de maneira negativa na organização e na gestão dos processos de trabalho da Saúde Bucal na Estratégia de Saúde da Família no Distrito Federal.

\section{Referência}

1. Starfield, B. Atenção primária: equilíbrio entre necessidades de saúde, serviços e tecnologia. Brasília: UNESCO, Ministério da Saúde, 2002. Disponível em: <http://bvsms.saude.gov.br/bvs/publicacoe s/atencao primaria p1.pdf>. Acesso em: 15 abr. 2019.

2. Fonseca Viegas SM, Mattos Penna CM. Integrality: life principle and right to health. Investigacion $\mathrm{y}$ educacion en enfermeria. 2015; 33(2): 237-47. Disponível em: <https://aprendeenlinea.udea.edu.co/revist
Processo de Trabalho na Estratégia de Saúde...

as/index.php/iee/article/view/23004/19238 >. Acesso em: 02 fev. 2018.

3. Baldani $\mathrm{MH}$ et al. A inclusão da odontologia no Programa Saúde da Família no Estado do Paraná, Brasil Inclusion of oral health services in the Family Health Program in the State of Paraná. Brazil. Cad Saude Publica. 2005, 21 (4): 1026-35. Disponível em: <https://www.nescon.medicina.ufmg.br/bi blioteca/imagem/0343.pdf >. Acesso em: 15 abr. 2019.

4. Narvai PC. Recursos humanos para promoção da saúde bucal: um olhar no início do século XXI. ABOPREV: promoção de saúde bucal. 2003, 3: 475-94. Disponível em: <http://www.fo.usp.br/wpcontent/uploads/QRecursoshumanos.pdf $>$. Acesso em: 2 fev. 2018.

5. Brasil. Portaria $n^{\circ} 2.436$, de 21 de setembro de 2017. Aprova a Política Nacional de Atenção Básica, estabelecendo a revisão de diretrizes para a organização da Atenção Básica, no âmbito do Sistema Único de Saúde (SUS). Diário Oficial da República Federativa do Brasil, Brasília, 21 set. 2017.

6. Brasil. Ministério da Saúde. Programa Nacional de Melhoria do Acesso e da Qualidade da Atenção Básica (PMAQ). Instrumento de Avaliação Externa para as Equipes de Atenção Básica.Brasília: Ministério da Saúde, 2013. Disponível em: <http://189.28.128.100/dab/docs/portaldab /documentos/instrumento_ae sfp.pdf $>$. Acesso em: 03 fev. 2018.

7. Brasil. Ministério da Saúde. Autoavaliação para a melhoria do acesso e da qualidade da atenção básica - AMAQ. Brasília: Ministério da Saúde, 2017. Disponível em: <http://189.28.128.100/dab/docs/portaldab /publicacoes/amaq_2017.pdf $>$ Acesso em: 03 fev. 2018.

8. Reis CDCL, Hortale VA. Programa Saúde da Família: supervisão ou" convisão"? Estudo de caso em município de médio 
Revista Eletrônica Gestão \& Saúde ISSN: 1982-4785 Moura RC, Souza TAC

porte. Cadernos de Saúde Pública. 2004, 20: $492-501$

9. Evans JR, Mathur A. The value of online surveys. Internet research. 2005, 15 (2): 195-219. Disponível em: $<$ https://pdfs.semanticscholar.org/f8de/bbb 585606d503f1049cfba3c1ddbad9352a8.pd f>. Acesso em: 10 fev. 2018.

10. Narvai PC. Odontologia e saúde bucal coletiva. São Paulo: Hucitec; 1994.

11. Moura LR et al. A participação do cirurgião-dentista no planejamento da Equipe Saúde da Família no município de Florianópolis, SC. Florianópolis: Universidade Federal de Santa Catarina; 2017. Disponível em:

<https://repositorio.ufsc.br/handle/123456 789/176566>. Acesso em: 03 fev. 2018

12. Brasil. Ministério da Saúde. Secretaria de Atenção à Saúde. Departamento de Atenção Básica. Cadernos de Atenção Básica; 17. Brasília: Ministério da Saúde, 2008. Disponível em:

<http://bvsms.saude.gov.br/bvs/publicacoe s/saude bucal.pdf $>$. Acesso em: $03 \mathrm{fev}$. 2018.

13. Rodrigues AAAO et al. Saúde bucal na Estratégia Saúde da Família em um município do semiárido baiano. Revista Baiana de Saúde Pública. 2012, 35 (3): 695. Disponível em: <http://files.bvs.br/upload/S/01000233/2011/v35n3/a2646.pdf>. Acesso em: 03 fev. 2018.

14. Da Cruz DB et al. Processo de trabalho na estratégia de saúde da família: uma perspectiva a partir da equipe de saúde bucal. Revista de APS. 2008, 12 (2). Disponível em:

<http://ojs2.ufjf.emnuvens.com.br/aps/arti cle/view/14291/7733>. Acesso em: 19 jan. $\underline{2018 .}$.

15. Pimentel FC et al. Caracterização do processo de trabalho das equipes de saúde bucal em municípios do Estado de Pernambuco, Brasil, segundo porte
Processo de Trabalho na Estratégia de Saúde...

populacional: da articulação comunitária à organização do atendimento clínico. Cad Saude Publica. 2012, 28 Supl: 146-57. Disponível em:

<http://www.scielo.br/pdf/csp/v28s0/15.pd f>. Acesso em: 19 jan. 2018.

16. Almeida GCM, Ferreira MAF. Saúde bucal no contexto do Programa Saúde da Família: práticas de prevenção orientadas ao indivíduo e ao coletivo. Cadernos de Saúde pública. 2008, 24: 2131-40. Disponível em: <http://www.scielo.br/pdf/csp/v24n9/19.pd f>. Acesso em: 12 abr. 2019.

17. Assis MMA, Jesus WLA. Acesso aos serviços de saúde: abordagens, conceitos, políticas e modelo de análise. Ciência \& Saúde Coletiva. 2012, 17: 2865-75. Disponível em : <http://www.scielo.br/pdf/csc/v17n11/v17 n11a02.pdf $>$. Acesso em: 03 fev. 2018.

18. Distrito Federal. Portaria $n^{\circ} .77$, de 14 de fevereiro de 2017. Estabelece a Política de Atenção Primária à Saúde do Distrito Federal. Diário Oficial do Distrito Federal, Brasília, 14 fev. 2017.

\section{Participação dos autores:}

MOURA, RC trabalhou na concepção teórica, coleta de dados, análise estatística e elaboração e redação final do texto.

SOUZA, TAC trabalhou na concepção teórica, elaboração e redação final do texto.

Recebido: 30.01 .2019

Revisado: 13.03.2019

Aprovado: 06.05.2019 